\title{
Construction of Hierarchical Multi-Organ Statistical Atlases and Their Application to Multi-Organ Segmentation from CT Images
}

\author{
Toshiyuki Okada ${ }^{1,2}$, Keita Yokota ${ }^{1,2}$, Masatoshi Hori ${ }^{3}$, Masahiko Nakamoto ${ }^{2,1}$, \\ Hironobu Nakamura ${ }^{3}$, and Yoshinobu Sato ${ }^{2,1}$ \\ ${ }^{1}$ Graduate School of Information Science and Technology, Osaka University, \\ ${ }^{2}$ Division of Image Analysis, \\ ${ }^{3}$ Department of Radiology, \\ Graduate School of Medicine, Osaka University, Suita, Osaka 565-0871, Japan
}

\begin{abstract}
Hierarchical multi-organ statistical atlases are constructed with the aim of achieving fully automated segmentation of the liver and related organs from computed tomography images. Constraints on inter-relations among organs are embedded in hierarchical organization of probabilistic atlases (PAs) and statistical shape models (SSMs). Hierarchical PAs are constructed based on the hierarchical nature of interorgan relationships. Multi-organ SSMs (MO-SSMs) are combined with previously proposed single-organ multi-level SSMs (ML-SSMs). A hierarchical segmentation procedure is then formulated using the constructed hierarchical atlases. The basic approach consists of hierarchical recursive processes of initial region extraction using PAs and subsequent refinement using ML/MO-SSMs. The experimental results show that segmentation accuracy of the liver was improved by incorporating constraints on inter-organ relationships.
\end{abstract}

\section{Introduction}

Statistical atlases, which represent anatomical variations among individuals, have been shown to be useful for segmentation and quantification of medical images [1]. In previous work, statistical atlases have typically been constructed for a single organ in a uniform manner [1. However, multiple organs are interrelated in the human body. Furthermore, the hierarchical nature is involved in the shape of a single organ, as well as multi-organ inter-relationships. Our previous study developed hierarchically decomposed statistical shape models (SSMs) 2]. Although the developed models were shown to be useful for accurate segmentation due to the hierarchical nature, the results in 2] clarified that explicit incorporation of the constraints of adjacent organs is essential to further improve accuracy. Statistical modeling of multi-organ structures will thus provide useful information for robust segmentation and shape recovery from medical images. Recent work [3] has tried to incorporate inter-organ relationships into SSMs. The basic limitation, however, is that quite large variations need to be statistically modeled for inter-organ relationships compared with a single organ. A quite large number of learning datasets

D. Metaxas et al. (Eds.): MICCAI 2008, Part I, LNCS 5241, pp. 502 509 2008.

(C) Springer-Verlag Berlin Heidelberg 2008 
are needed to model complex structures. Due to this limitation, previous works limited applications to $2 \mathrm{D}$ or simple $3 \mathrm{D}$ shapes 3 .

In this paper, we embed the constraints for multi-organ inter-relationships into hierarchically organized statistical atlases to deal with the large variations involved. Hierarchical organization schemes have been developed for two atlas representations: probabilistic atlases (PAs) 4 and SSMs [1. Spatial normalization for constructing and utilizing PAs is progressively performed based on the predefined hierarchy of organ structures. SSMs are also hierarchically constructed within single organ shape, as well as across multiple organs. Sub-shapes within a single organ shape or sub-shapes across multiple organ shapes are hierarchically organized. By restricting SSM construction to local sub-shapes in inter-related organs rather than to whole shapes, variations in multi-organ inter-relationships are effectively modeled using a moderate number of training datasets. Further, multiorgan SSMs of whole shapes are realized by hierarchical organization of SSMs constructed for decomposed sub-shapes. Hierarchy in constructed PAs and SSMs is directly linked to a hierarchical automated segmentation procedure, where hierarchically normalized PAs are utilized to provide good initial conditions for subsequent multi-organ SSM fitting. We experimentally evaluated the effects of integrating multi-organ inter-relationships on performance improvements in segmentation results of the liver from computed tomography (CT).

\section{Methods}

\subsection{Hierarchical Probabilistic Atlas}

Given hierarchical relationships of inter-related organ structures, spatial normalization is performed according to the given hierarchy. Here, we considered the hierarchy of the abdominal cavity, liver, vena cava and gallbladder (Fig. 1). Shape and position of the anatomical structures of lower hierarchy levels, e.g., the gallbladder and vena cava, are strongly constrained by those of higher hierarchy levels, e.g., the liver. Spatial normalization for an organ of interest is performed by mapping the dataset for an individual patient into the normalized space through nonrigid registration [5] using the organ shape of the next highest hierarchy level to remove unwanted shape and positional variations as well as represent datasets in the canonical frame (left side of Fig. 2).

Before the PA is constructed, we assume that the regions of each anatomical structure have already been segmented from the CT datasets. In the case of normalization for the gallbladder and vena cava, a dense three-dimensional (3D) deformation field is obtained by nonrigid registration of individual liver shapes to the average liver shape. Gallbladder and vena cava regions are normalized based on the average liver shape by warping individual CT datasets using the obtained deformation fields. The PA is constructed by averaging the warped segmented binary images of each structure. The right side of Fig. 2 shows constructed PAs of the gallbladder and vena cava normalized using the liver in comparison with those using the abdominal cavity and those without normalization. The 
high-probability area (colored red) was increased in the liver-normalized PA, showing high predictive performance.

We refer to the hierarchy described in this subsection as "inter-organ hierarchy", to differentiate from "intra-organ hierarchy" described in the next section.

\subsection{Hierarchical Organization of Multi-Organ Statistical Shape Models}

We considered two inter-related organ shapes. Let $S$ (e.g., the liver) and $T$ (e.g., the vena cava or gallbladder) be the sets of vertices comprised in the surface models of the two shapes. All training datasets of $S$ and $T$ are nonrigidly registered to the surface model of the standard shape of $S$ and $T$, respectively [5], so that they have the same topology of the vertices as the standard shape. That is, correspondences of all vertices are known among the datasets. Let $S^{\prime}$ and $T^{\prime}$ be the sets of vertices of the sub-shapes (hereafter called "patches") of $S$ and $T$, respectively. Let $U$ be the union of $S^{\prime}$ and $T^{\prime}$. We defined multi-organ SSM (MO-SSM) as the SSM of $U$.

A multi-level SSM (ML-SSM) 2] of $S$ is defined as the set of SSMs of hierarchically decomposed patches $S_{i}$ and $S_{i j}$, which denote all patches and the $j$-th patch of "intra-organ hierarchy" level $i$, respectively, where $i=0,1, \cdots m$. As correspondences of all vertices among datasets are known, these decompositions can be determined for all datasets automatically if the decomposition is defined only for the standard shape dataset. $T_{i}$ and $T_{i j}(i=0,1, \cdots, n)$ are defined similarly. Principal component analysis is performed for each patch.

In constructing MO-SSM, we assume that the set of the patches of $U\left(=S^{\prime} \cup\right.$ $\left.T^{\prime}\right)$ satisfy the conditions, $S^{\prime} \subseteq S_{n}$ and $T^{\prime} \subseteq T_{m}$, that is, $U \subseteq\left(S_{n} \cup T_{m}\right)$. Figure 3 shows an example of MO-SSM, where $S$ and $T$ are the liver and gallbladder (or vena cava), respectively, $m=2$, and $n=0$. The patches on $S$ (the liver), which are closely inter-related to $T$ are selected as $S^{\prime}$, while the whole shape of the gallbladder (or vena cava), that is $T_{0}$, is selected as $T^{\prime}$. In this case, two patches on $S$ at intra-organ hierarchy level 2 are selected for $S^{\prime}$, at least one vertex of which is located within the pre-determined distance to the surface of $T$ through all datasets.

To connect two ML-SSMs for different organs with MO-SSM, the adhesiveness constraints 2] are combined. Adhesiveness constraint was originally introduced to remove inconsistencies in adjacent patches at the same hierarchy level in ML-SSM. The decomposition of patches is performed so that adjacent patches overlap each other along the boundaries. The adhesive constraint ensures that two adjacent patches adhere to each other in the overlapped area to recover the consistent whole single shape. MO-SSM of $U$ overlaps with $S$ and $T$ only on patches $S^{\prime}$ and $T^{\prime}$, respectively. The adhesive constraint is applied to the overlap areas $S^{\prime}$ and $T^{\prime}$ so that the sum of the distances between vertices of MO-SSM for $U$ and the corresponding vertices for $S^{\prime}$ or $T^{\prime}$ in ML-SSM of $S$ or $T$, respectively, is sufficiently small. As the MO-SSM is limited to local region $S^{\prime}$ rather than the whole shape of $S$ (the liver in this case), the involved variations can be efficiently encoded even without a large number of training datasets. 


\subsection{Hierarchical Approach to Multi-Organ Segmentation from CT}

Based on the constructed hierarchical PAs and ML/MO-SSMs, a hierarchical automated segmentation procedure is derived by assuming that anatomical structure at inter-organ hierarchy level 0 has already been extracted. In this paper, we assumed that the anatomical structure at level 0 is the approximated abdominal cavity region (as shown in Fig. 1 and Fig. 2), which is extracted based on the lung and bone regions. We confirmed that the approximated abdominal cavity region can be reliably extracted using more than $100 \mathrm{CT}$ datasets.

The basic approach is as follows:

Step 0: Inter-organ hierarchy level $k \leftarrow 0$.

Step 1: Region extraction of anatomical structures at inter-organ hierarchy level $k+1$ and refinement of those extracted at level $k$, in which datasets are spatially normalized using anatomical structures obtained at level $k$ or higher (a smaller number means higher level).

Step 1-1: Initial region extraction using PAs 2] 6].

Step 1-2: Refinement of extracted regions of structures at level $k+1$ using ML-SSMs 2, wherein intra-organ hierarchy is embedded (only when $k=0$ in the experiments).

Step 1-3: Refinement of extracted regions of structures at levels $k$ and $k+1$ using MO-SSMs (only when $k=1$ in the experiments).

Step 2: $k \leftarrow k+1$. Go to Step 1 .

In Step 1-1, likelihood based on position and intensity is estimated at each voxel position from PA and CT data, then voxel-based segmentation is performed [6]. In Step 1-2, ML-SSM is successively fitted to refine the segmentation. The details of these methods are described in 2 6]. In this paper, we have added new Step 1-3 for refinement of segmentation results of anatomical structures at levels $k+1$ and $k$ using the inter-organ constraints.

Let $\mathbf{s}^{\prime}$ and $\mathbf{t}^{\prime}$ be the shape parameter (coefficient) vector of $S^{\prime}$ and $T^{\prime}$ in single organ ML-SSMs of $S$ and T, respectively, and $\mathbf{u}^{\prime}$ be that of the MO-SSM of $U$ $\left(S^{\prime}\right.$ and $T^{\prime}$ ). Let $P_{S}$ and $P_{T}$ be the sets of detected edge points for $S$ and $T$ from $\mathrm{CT}$ data, respectively. The detection method for edge points is described in 11. Given $P_{S}$ and $P_{T}$, we estimate the shape parameter vector $\mathbf{s}^{\prime}, \mathbf{t}^{\prime}$, and $\mathbf{u}^{\prime}$ by minimizing

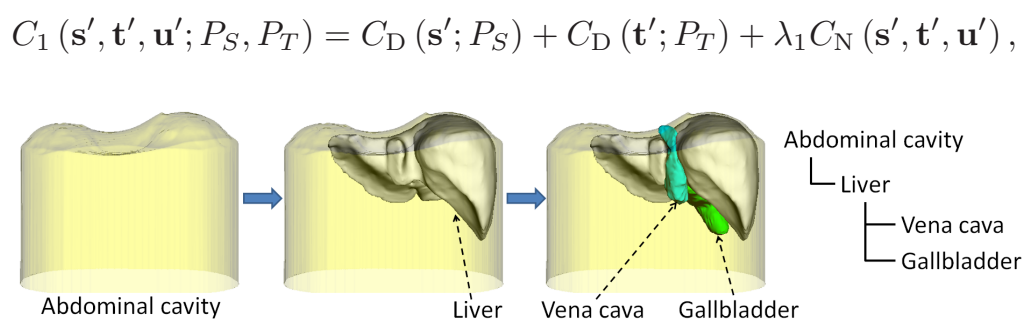

Fig. 1. Hierarchy in inter-organ relationships of the abdominal cavity, liver, vena cava and gallbladder 


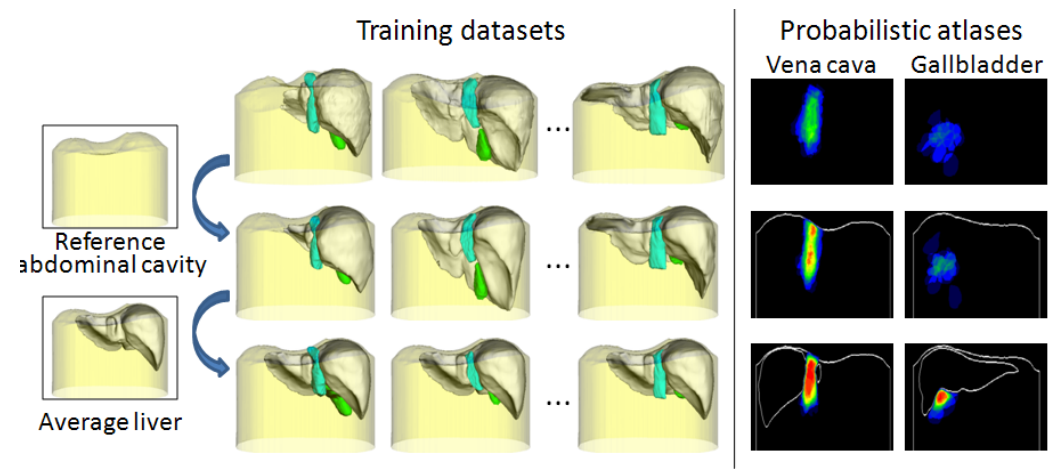

Fig. 2. Hierarchical spatial normalization (left) and resulting probabilistic atlases (right). Upper row: Original datasets. Middle row: Normalized datasets by the abdominal cavity. Lower row: Normalized datasets by the liver. In the liver-normalized space, probabilistic atlases have high probability area (colored red) compared with other probabilistic atlases having relatively wide low probability areas (colored blue).

where $C_{\mathrm{D}}\left(\mathbf{s}^{\prime} ; P\right)$ is the sum of distances between model surface $S^{\prime}$ and edge points $P$, and $C_{\mathrm{N}}\left(\mathbf{s}^{\prime}, \mathbf{t}^{\prime}, \mathbf{u}^{\prime}\right)$ is the inter-organ constraint. $\lambda_{1}$ is a weight parameter balancing these constraints. The edge detection process and minimization process of Eq. (11) are repeatedly performed. After this, final refinement is performed. Let $\mathbf{s}^{\prime \prime}$ be a shape parameter vector of $\mathbf{s}$ but not included in $\mathbf{s}^{\prime}$ and $R$ be the estimated shape by minimization Eq. (1). As a final process of segmentation, by fixating $\mathbf{s}^{\prime}$ obtained by minimizing Eq. (1), we estimate the remaining shape parameters $\mathbf{s}^{\prime \prime}$ by minimizing

$$
C_{2}\left(\mathbf{s}^{\prime \prime} ; R\right)=C_{\mathrm{D}}\left(\mathbf{s}^{\prime \prime} ; R\right)+\lambda_{2} C_{\mathrm{A}}\left(\mathbf{s}^{\prime \prime}\right),
$$

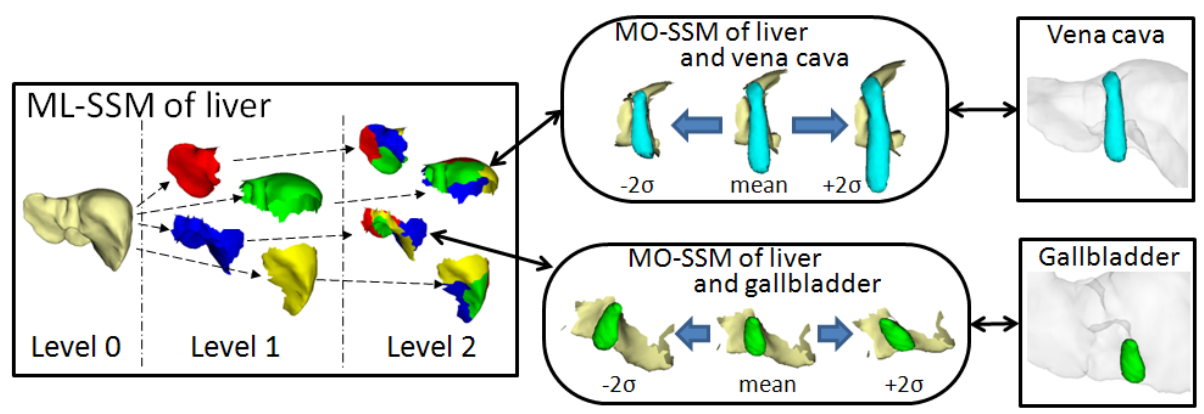

Fig. 3. Hierarchical multi-organ statistical shape models (MO-SSMs). In this case, an MO-SSM (middle) is constructed for the union of small local patches of the liver and the whole shape of the vena cava or gallbladder. 
where $C_{\mathrm{D}}\left(\mathbf{s}^{\prime \prime} ; R\right)$ is the sum of distances between the current model surface and $R$, and $C_{\mathrm{A}}\left(\mathbf{s}^{\prime \prime}\right)$ is the adhesiveness constraint for overlap regions to eliminate inconsistency among adjacent patches [2] $\lambda_{2}$ is a weight parameter balancing the two constraints.

\section{Results}

Twenty-eight abdominal CT datasets (slice thickness, $2.5 \mathrm{~mm}$; pitch, $1.25 \mathrm{~mm}$; Field of view $(\mathrm{FOV}) 350 \times 350 \mathrm{~mm}^{2}, 512 \times 512$ matrix, 159 slices) were used. Contrast agent was used for CT. We randomly selected 8 datasets for evaluation, and others for training. The hierarchical PA and ML-SSMs for the liver, vena cava and gallbladder, and MO-SSMs of liver-vena cava and liver-gallbladder were constructed from the 20 training datasets. In ML-SSMs for the vena cava and gallbladder, the number of intra-organ hierarchy levels was 1 , thus representing just conventional SSMs. ML-SSM for the liver used in the experiments was the same as described in [2]. We used $\lambda_{1}=2.0$ and $\lambda_{2}=0.4$ for the MO-SSMs of liver-vena cava and liver-gallbladder, respectively, which were experimentally determined. Segmentation of the liver was performed first, then the vena cava and gallbladder were segmented. Finally, segmentation results for these three organs were refined using MO-SSMs. Given the CT datasets input for evaluation, all procedures were performed in a fully automated manner according to the basic approach described in the previous subsection.

Figures 4 (a) and (b) show the results of segmentation accuracy of the liver around the vena cava and gallbladder, respectively. Absolute surface distance (ASD) [7] was used as a measure of segmentation accuracy. Manually traced regions were used for reference. By combining MO-SSMs of liver-vena cava and liver-gallbladder, accuracy was improved in all cases compared with using the ML-SSM of the liver alone (on average from $2.69 \mathrm{~mm}$ to $2.04 \mathrm{~mm}$ around the vena cava and from $2.51 \mathrm{~mm}$ to $1.87 \mathrm{~mm}$ around the gallbladder). Figure 5 shows segmentation results for two illustrative cases. In Fig. 5 (a), MO-SSM of the liver

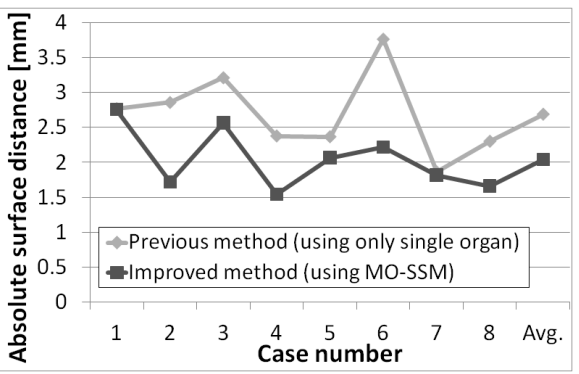

(a)

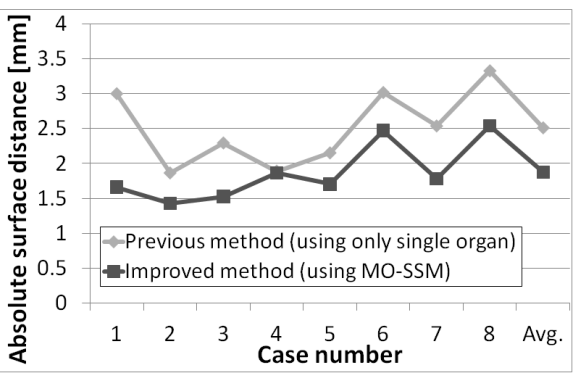

(b)

Fig. 4. Evaluation results of segmentation accuracy of the liver around (a) the vena cava and (b) gallbladder 


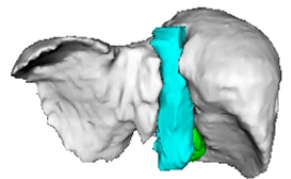

True shapes

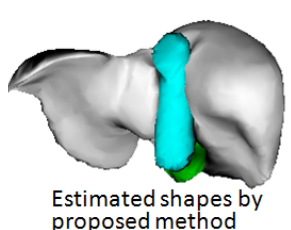
proposed method

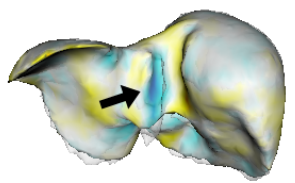

Estimated liver shape by previous method $(89.7 \% / 1.20 \mathrm{~mm})$

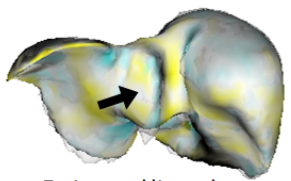

Estimated liver shape by proposed method (90.7\% / $1.05 \mathrm{~mm})$

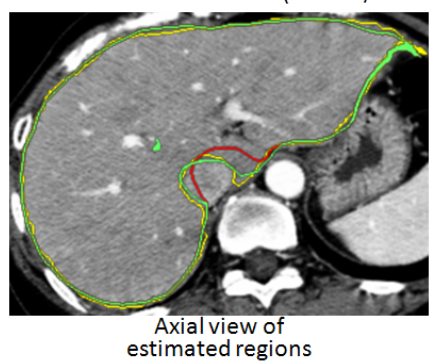

(a)

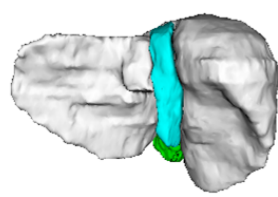

True shapes

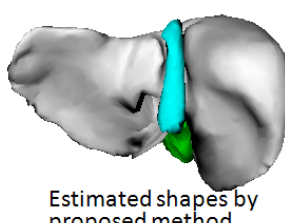

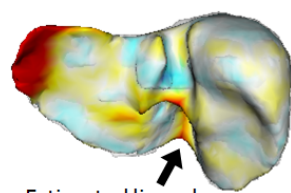

Estimated liver shape by previous method $(87.8 \% / 1.62 \mathrm{~mm})$

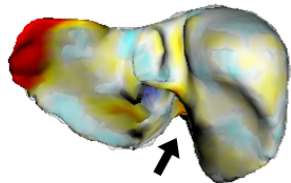

Estimated liver shape by proposed method $(88.5 \% / 1.49 \mathrm{~mm})$

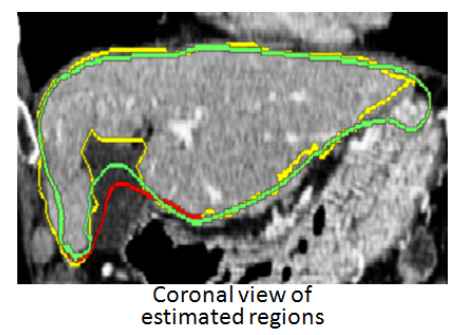

(b)

Fig. 5. Results of illustrative cases. (a) Case 2. MO-SSM of the vena cava was effective in this case (shown by arrow). (b) Case 8. MO-SSM of the gallbladder was effective in this case (shown by arrow). Estimated liver shapes are shown as color codes representing signed surface distance errors (red, convex error; white, no error; blue, concave error). Typical CT cross-sections are also shown (red contour, estimated by previous method; green, proposed method; yellow, ground truth). Note that unshown parts of the red contours overlap with the green contours.

and vena cava was effective and the segmentation result was improved around the vena cava. In Fig. 5 (b), similar improvements were observed around the gallbladder. The proposed method improves the segmentation accuracy only for local regions around the vena cava and gallbladder. By averaging over the whole liver, the improvements become superficially small and ASD was improved from 1.59 to $1.46 \mathrm{~mm}$ and volumetric overlap (VO) [7] from 87.9 to $88.8 \%$.

\section{Discussion and Conclusions}

We have described construction of hierarchical multi-organ statistical atlases and application of this method to segmentation of the liver and other peripheral organs (vena cava and gallbladder) from CT. Using multi-organ statistical atlases, segmentation accuracy of the liver was improved. In particular, segmentation accuracy around the caudate lobe located near the vena cava was significantly 
improved. The caudate lobe is a small but clinically important liver lobe, and semi-automated segmentation is considered difficult [8]. Improved segmentation accuracy around the caudate lobe is thus worthy of note.

The methods described in this paper were performed in a fully automated manner. Hierarchically organized PAs were particularly useful for automation. The initial regions of the vena cava and gallbladder were effectively extracted using PAs normalized by the average liver. Without hierarchical normalization, automation would often fail. Hierarchical organization of PAs and MO/ML-SSMs is directly linked to the hierarchical segmentation procedure.

In this work, we constructed multi-organ statistical atlases for the vena cava and gallbladder for modeling inter-relationships with the liver. However, other organs, such as the stomach, heart, and kidney, are inter-related with the liver in addition to the vena cava and gallbladder. As shown in Fig. 5 (a), large segmentation error was observed in the left lobe in two out of eight cases. This error was caused by ambiguity in boundaries of the liver and stomach. Similar error was observed at boundaries of the liver and heart in one case. Since we used contrast CT images, the boundaries of the liver and kidney were clear, and segmentation error was not observed at the boundaries. We considered that the vena cava and gallbladder are strongly constrained by the liver rather than the stomach and heart, and were incorporated into MO-SSMs first. More organs can be incorporated using the proposed method to improve segmentation accuracy of the liver. As future work, we plan to incorporate other organs, such as the stomach and heart.

\section{References}

1. Lamecker, H., et al.: Segmentation of the liver using a 3D statistical shape model. Technical report, Zuse Institue, Berlin (2004)

2. Okada, T., et al.: Automated segmentation of the liver from $3 \mathrm{D}$ ct images using probabilistic atlas and multi-level statistical shape model. In: Proc. Medical Image Computing and Computer-Assisted Intervention, pp. 86-93 (2007)

3. Yang, J., et al.: Neighbor-constrained segmentation with level set based 3-D deformable models. IEEE Transactions on Medical Imaging 34(8), 940-948 (2004)

4. Park, H., et al.: Construction of an abdominal probabilistic atlas and its application in segmentation. IEEE Transactions on Medical Imaging 22(4), 483-492 (2003)

5. Chui, H., et al.: A new point matching algorithm for non-rigid registration. Computer Vision and Image Understanding 89, 114-141 (2003)

6. Zhou, X., et al.: Constructing a probabilistic model for automated liver region segmentation using non-contrast X-ray torso CT images. In: Proc. Medical Image Computing and Computer-Assisted Intervention, pp. 856-863 (2006)

7. Heimann, T., et al.: MICCAI workshop on 3D segmentation in the clinic (2007), http://mbi.dkfz-heidelberg.de/grand-challenge2007/

8. Hermoye, L., et al.: Liver segmentation in living liver transplant donors: Comparison of semiautomatic and manual methods. Radiology 234(1), 171-178 (2005) 